\section{Effect of Educational Interventions to Reduce Readmissions due to Heart Failure Decompensation in Adults: a Systematic Review and Meta-analysis}

\author{
Wilson Cañon-Montañez $z^{1,4}$ \\ https://orcid.org/0000-0003-0729-5342 (DD \\ Tatiana Duque-Cartagena ${ }^{2,4}$ \\ https://orcid.org/0000-0003-0881-1441 (iD) \\ Alba Luz Rodríguez-Acelas 3,4 \\ https://orcid.org/0000-0002-7384-3522 (D)
}

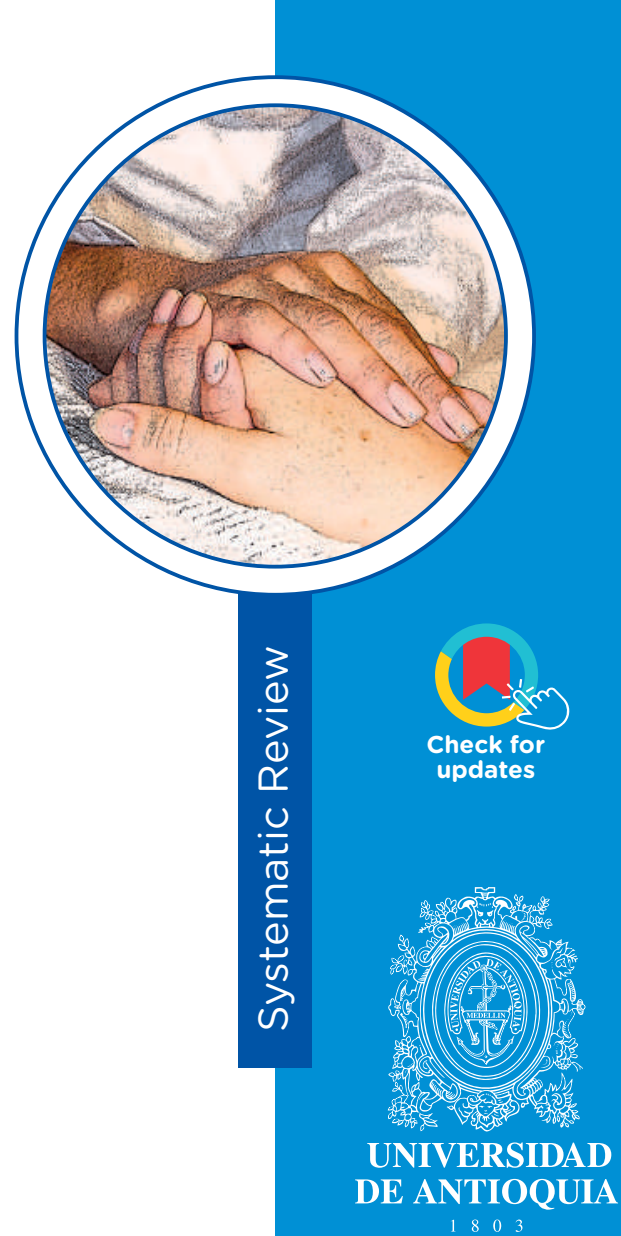

1 RN, Ph.D. Associate Professor.

Email: wilson.canon@udea.edu.co. Corresponding author.

2 Undergraduate nursing student, young researcher. Email: tatiana.duquec@udea.edu.co

3 RN, Ph.D. Associate Professor. Email: aluz.rodriguez@udea.edu.co

4 Faculty of Nursing, Universidad de Antioquia. MedeIlín, Colombia.

Conflicts of interest: None.

Received: February 23, 2021.

Approved: May 10, 2021.

How to cite this article: Cañon-Montañez W, DuqueCartagena T, Rodríguez-Acelas AL. Effect of Educational Interventions to Reduce Readmissions due to Heart Failure Decompensation in Adults: a Systematic Review and Meta-analysis. Invest. Educ. Enferm. 2021; 39(2): e05.

DOI: https://doi.org/10.17533/udea.iee.v39n2e05.

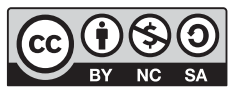

https://creativecommons.org/licenses/by-nc-sa/4.0/ 
was evaluated through the inconsistency statistic $\left(I^{2}\right)$. Results. Of 2369 studies identified, 45 were included in the SR and 43 in the MA. The MA of studies with follow-up at six months showed a decrease in readmissions of 30\% (RR: $0.70 ; 95 \%$ $\mathrm{Cl}: 0.58$ to $0.84 ; I^{2}: 0 \%$ ) and the 12 -month follow-up evidenced a reduction of $33 \%$ (RR: $0.67 ; 95 \% \mathrm{Cl}: 0.58$ to $0.76 ; \mathrm{I}^{2}: 52 \%$ ); both analyses in favor of the El group. Regarding the time of hospital stay, a reduction was found of approximately two days in patients who received the El (MD: $-1.98 ; 95 \% \mathrm{Cl}:-3.27$ to $-0.69 ; \mathrm{I}^{2}: 7 \%$ ). Conclusion. The findings support the benefits of El to reduce readmissions and days of hospital stay in adult patients with heart failure.

Descriptors: heart failure; patient readmission; patient education as topic; self-care; systematic review.

\section{Efecto de intervenciones educativas para reducir readmisiones debido a descompensación de falla cardiaca en adultos: una revisión sistemática y meta- análisis}

\section{Resumen}

Objetivo. Estimar el efecto combinado de las intervenciones educativas (IE) en la disminución de readmisiones y tiempo de estancia hospitalaria en adultos con falla cardiaca comparado con el cuidado usual. Métodos. Revisión Sistemática (RS) y meta-análisis (MA) de ensayos clínicos aleatorizados que siguieron las recomendaciones de la declaración PRISMA. El protocolo se registró en PROSPERO (CRD42019139321). Se realizaron búsquedas desde el inicio hasta julio de 2019, en las bases de datos PubMed/Medline, Embase, Cochrane CENTRAL, Lilacs, Web of Science y Scopus. El MA se realizó mediante modelo de efectos aleatorios. La medida de efecto utilizada para los desenlaces dicotómicos fue el riesgo relativo (RR) y para desenlaces continuos se usó la diferencia de medias (DM), con sus intervalos de confianza (IC) del 95\%. La heterogeneidad se evaluó mediante el estadístico de inconsistencia $\left(\mathrm{I}^{2}\right)$. Resultados. De 2369 estudios identificados, 45 se incluyeron en la RS y 43 en el MA. El MA de estudios con seguimiento a seis meses mostró una disminución en las readmisiones de 30\% (RR: 0.70; IC 95\%: 0.58 a 0.84; $I^{2}$ : 0\%) y el seguimiento a doce meses evidenció una reducción de 33\% (RR: 0.67; IC 95\%: 0.58 a $0.76 ; I^{2}: 52 \%$ ), ambos análisis a favor del grupo de IE. Referente al tiempo de estancia hospitalaria, se encontró una reducción de aproximadamente dos días 
en los pacientes que recibieron las IE (DM: - 1.98 ; IC 95\%: -3.27 a -0.69; I: 7\%). Conclusión. Los hallazgos soportan los beneficios de las IE para la disminución de readmisiones y días de estancia hospitalaria en pacientes adultos con falla cardiaca.

Descriptores: insuficiencia cardiaca; readmisión del paciente; educación del paciente como asunto; autocuidado; revisión sistemática.

\section{Efeito de intervenções educacionais para reduzir as readmissões devido à descompensação da insuficiência cardíaca em adultos: uma revisão sistemática e meta- análise}

\section{Resumo}

Objetivo. Estimar o efeito combinado de intervenções educacionais (IE) na redução de readmissões e tempo de internação em adultos com insuficiência cardíaca, em comparação com o cuidado usual. Métodos. Revisão sistemática (RS) e meta-análise (MA) de ensaios clínicos randomizados que seguiu as recomendações da declaração PRISMA. O protocolo foi registrado no PROSPERO (CRD42019139321). Foram realizadas buscas desde o início até julho de 2019, nas bases de dados PubMed/ Medline, Embase, Cochrane CENTRAL, Lilacs, Web of Science e Scopus. A MA foi realizada usando um modelo de efeitos aleatórios. A medida de efeito utilizada para desfechos dicotômicos foi o risco relativo (RR) e para desfechos contínuos foi usada a diferença de médias (DM), com seus intervalos de confiança (IC) de $95 \%$. A heterogeneidade foi avaliada por meio da estatística de inconsistência $\left(I^{2}\right)$. Resultados. De 2369 estudos identificados, 45 foram incluídos na RS e 43 na MA. A MA dos estudos com seguimento de seis meses mostrou uma diminuição nas readmissões de $30 \%$ (RR: 0.70; IC 95\%: 0.58 a 0.84; $I^{2}$ : 0\%) e o seguimento de doze meses mostrou uma redução de 33 \% (RR: 0.67; IC 95\%: 0.58 a 0.76; $I^{2}: 52 \%$ ), ambas as análises em favor do grupo de IE. Em relação ao tempo de internação, foi observada uma redução de aproximadamente dois dias nos pacientes que receberam as IE (DM: -1.98; IC 95\%: -3.27 a -0.69; I²: 7\%). Conclusão. Os achados evidenciam os benefícios das IE para a redução de readmissões e dias de internação em pacientes adultos com insuficiência cardíaca.

Descritores: insuficiência cardíaca; readmissão do paciente; educação de pacientes como assunto; autocuidado; revisão sistemática. 


\section{Introduction}

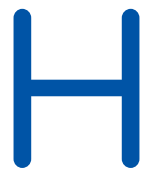

eart failure (HF) is part of the group of cardiovascular diseases. Defining this disease is complex, given that it involves different processes and its etiology is also varied, which is why it is referred to as a "syndrome". Simply stated, it may be understood as "state in which the heart is not capable to pump the amount of blood necessary to fulfil the needs of the organism". (1) Moreover, due to its high morbidity and mortality figures, ${ }^{(2,3)}$ currently, HF is considered a public health problem, besides implying a high cost for governments and health systems. Evidence shows that the prevalence of HF increases gradually with age and it is estimated to affect $10 \%$ of elderly adults, becoming the first cause of hospitalization in this population. ${ }^{(4)}$

In relation with the socioeconomic burden due to $\mathrm{HF}$, some European and South American countries show high costs for health services; ${ }^{(3,5,6)}$ which has become a great concern for the governments and health institutions. Another one of the serious problems of HF is the increase of readmissions of patients due to the decompensation of the disease. ${ }^{(4)}$ Within this context, over time, specialized units have been created with programs of multidisciplinary approach for the integral management of patients with HF. ${ }^{(3)}$ Among these programs, education of patients is crucial to improve the clinical outcomes of patients. Health education is one of the professional roles of nursing. Nurses must have the ability to evaluate the patients' individual needs for education and be able to improve their self-care practices that contribute to the reduction of readmissions. ${ }^{(2)}$ Educational interventions can vary in their intensity, methodology, or strategy. The effect sought with these interventions is to achieve a greater number of patients with HF aware of their disease and of the importance of self-care habits for their health. This, in turn, favors better control of the disease and reduction of the different complications and costs associated with $\mathrm{HF}^{(5,6)}$

Due to the aforementioned, up-to-date syntheses are required of the literature that evidences the effect the educational interventions have on reducing readmissions due to decompensation of the HF syndrome. Although primary studies exist to address this problem, it is important to group systematically every evidence to permit greater comprehension of the phenomenon and generate new results that contribute to the recovery of individuals who endure this disease. Hence, the objective of this study was to estimate the combined effect of the educational interventions on reducing hospital readmissions and time of hospital stay in adults with HF, compared with usual care. 


\section{Methods}

Design and registry of the protocol. This was a systematic review (SR) and meta-analysis (MA) of randomized controlled trials (RCTs) that followed the recommendations of the PRISMA (Preferred Reporting Items for Systematic Reviews and Meta-Analyses) statement ${ }^{(7)}$ and of the Cochrane Handbook ${ }^{(8)}$ for SR of intervention studies. The protocol was registered in the International Prospective Register of Systematic Reviews (PROSPERO) with code CRD42019139321.

Source of data and search strategy. The information was collected from the following electronic databases: PubMed/Medline, Embase, Lilacs, Cochrane CENTRAL, Scopus, and Web of Science. Searches were made from inception until July 2019, using MeSH terms and entry terms for PubMed/Medline, emtree terms for Embase and descriptors for the other databases. Likewise, the following filters were used for the search strategy: randomized controlled trials, studies in humans and English, Portuguese, Spanish languages. To identify additional studies, search was made in other sources that included the review of references of the studies included, SR published, and the network of primary registries of RCTs recognized by the World Health Organization.

The following search strategy was used for PubMed/Medline: ((Heart failure[MeSH Terms]) OR (Cardiac Failure)) OR (Heart Decompensation)) OR (Decompensation, Heart)) $O R$ (Congestive heart failure)) OR (Heart Failure, Congestive)) )) AND (Knowledge[MeSH Terms])) AND (Self-care[MeSH Terms]))) OR (Care, Self)) OR (Self-care behaviors[MeSH Terms])) OR (Self-management[MeSH Terms])) OR (Management, Self)) OR (Self-efficacy[MeSH Terms])) OR (Efficacy, Self)) OR (Self Concept[MeSH Terms])) OR (Self-confidence)) OR (Confidence, Self)) AND (Education[MeSH Terms])) AND (Patient education[MeSH Terms])
)) $O R$ (Education, Patient)) ) OR (Education of Patients) ) AND (Education, nursing [MeSH Terms])) ) OR (Nursing Education)) OR (Educations, Nursing)) OR (Nursing Educations)) AND (Health education[MeSH Terms])) OR (Education, Health)) AND (Standard of Care).

Eligibility criteria of the studies. This SR and MA included experimental studies or RCTstype intervention studies. The following PICO (population, intervention, comparator, outcomes) research question was used to consider the eligibility of the studies, P: adult patients with $\mathrm{HF}$ in any stage of the disease; I: educational interventions; C: usual or standard care, and O: reduced readmissions and time of hospital stay due to decompensation of the HF.

Data extraction. Identification and selection of the studies was performed independently by two reviewers, who were young undergraduate researchers with prior training and certification in SR and MA. Disagreements were solved through the intervention of a third reviewer, senior researcher with $\mathrm{PhD}$ formation and experience in SR and MA. Articles duplicated in several databases were considered only once. The Mendeley reference manager was used to store references and eliminate duplicate studies.

Outcomes. The principal outcome was the decrease of hospital readmissions due to decompensation of the HF and the secondary outcome was the decrease of days of hospital stay.

Evaluation of the risk of study bias. The risk of bias (RoB 1) tool from the Cochrane Collaboration (9) was used to evaluate the risk of bias in RCTs. The following parameters were evaluated: random sequence generation and allocation concealment, blinding of participants and personnel, blinding of outcome assessment, incomplete outcome data, selective reporting of results and other sources of bias.

Data analysis. Estimation of the grouped effect was conducted with the Review Manager (RevMan 5.4) software from the Cochrane Collaboration. 
The dichotomous results are presented and compared by using relative risk (RR) through the Mantel-Haenszel method and for continuous results the mean difference (MD) is presented through the inverse-variance weighted; both with their respective 95\% confidence intervals (Cl). Likewise, to quantify the heterogeneity of the studies included, the inconsistency $\left(I^{2}\right)$ statistic was used and the graphic presentation of the MA results used the forest plot. To evaluate publication bias or bias due to missing results, the Stata 16.0 software was used, through the Egger test and the funnel plot.

\section{Results}

\section{Identification and selection of the studies}

The work identified 2369 studies, of which 45 studies were included in the SR and data from 43 studies were included in the MA. Two studies were excluded from the MA because the data on readmissions corresponded to follow-up times different from the other studies and, hence, it was not possible to meta-analyze. The flow diagram for the selection and exclusion of studies is shown in Figure 1.
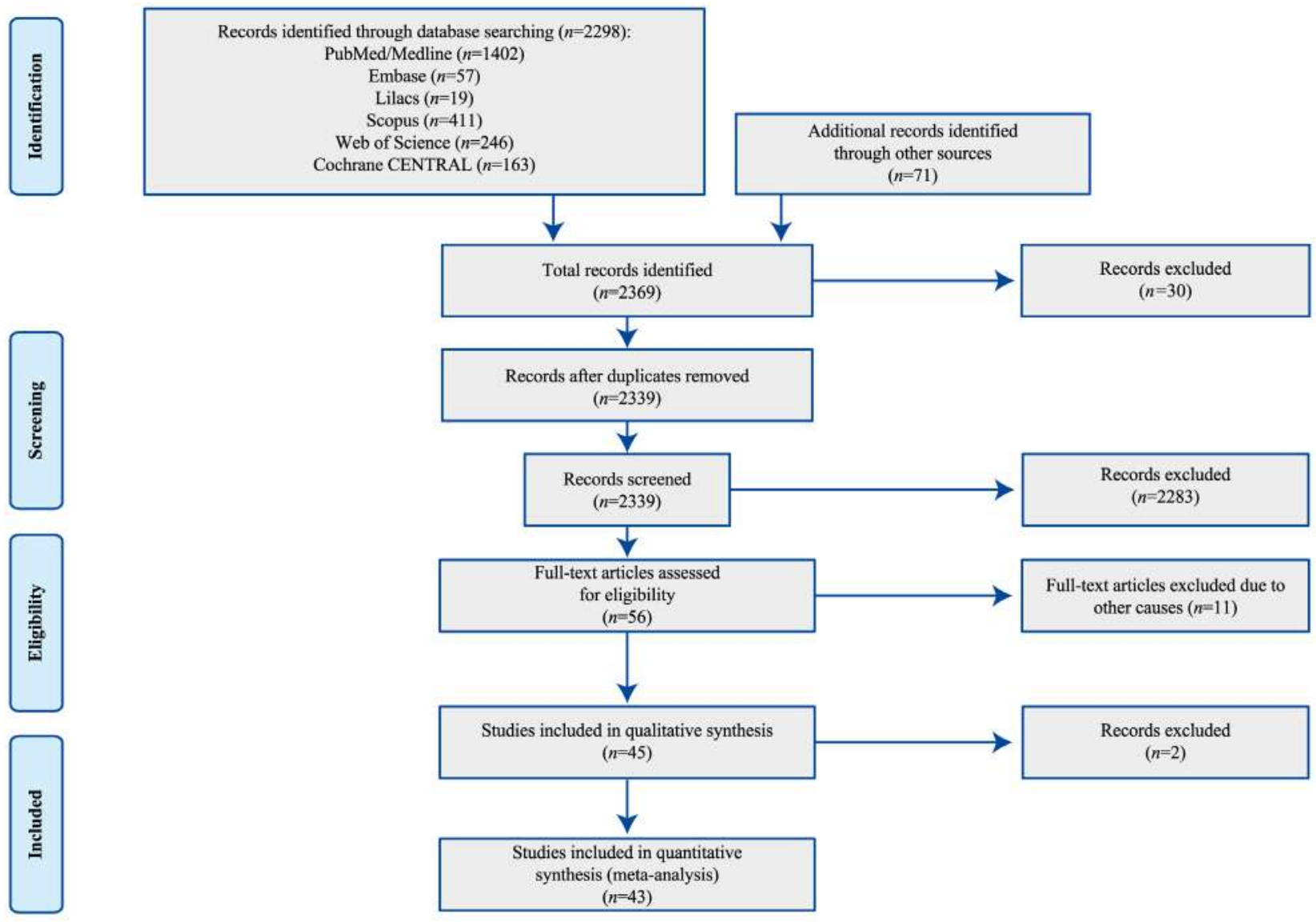

Figure 1. PRISMA flow diagram for the studies selection 
Characteristics of studies included

The general description of the studies is shown in Table 1, which contains the author, year of publication, country, a brief description of the intervention, time of follow-up, and most relevant results for the research.

\section{Table 1. Characteristics of the included studies}

\begin{tabular}{|c|c|c|c|c|c|c|}
\hline $\begin{array}{l}\text { First } \\
\text { author, } \\
\text { year }\end{array}$ & Country & $\begin{array}{l}\text { Sam- } \\
\text { ple } \\
\text { size }\end{array}$ & Intervention group & Follow-up & $\begin{array}{l}\text { Control } \\
\text { group }\end{array}$ & Main outcomes \\
\hline $\begin{array}{l}\text { Aldamiz- } \\
\text { Echevarría } \\
\text { et al., } \\
2007 \text { (10) }\end{array}$ & Spain & 279 & $\begin{array}{l}\text { Educational program on basic data } \\
\text { of } \mathrm{HF} \text { and its treatment. }\end{array}$ & $\begin{array}{l}3,6 \text { and } 12 \\
\text { months }\end{array}$ & $\begin{array}{l}\text { Standard } \\
\text { care }\end{array}$ & $\begin{array}{l}\text { Readmissions* at } 12 \\
\text { months } \\
\text { Intervention: } 55 \\
\text { Control: } 57 \\
\text { Days of hospital stay+ } \\
\text { Intervention: } 8.5(6.4) \\
\text { Control: } 8.4(11.6)\end{array}$ \\
\hline $\begin{array}{l}\text { Atienza et } \\
\text { al., } 2004 \\
(11)\end{array}$ & Spain & 338 & $\begin{array}{l}\text { Education before discharge on } \\
\text { knowledge of the disease and its } \\
\text { management. Home visits. }\end{array}$ & 12 months & $\begin{array}{l}\text { Standard } \\
\text { care }\end{array}$ & $\begin{array}{l}\text { Readmissions* at } 12 \\
\text { months } \\
\text { Intervention: } 61 \\
\text { Control: } 122\end{array}$ \\
\hline $\begin{array}{l}\text { Blue et al., } \\
2001 \\
(12)\end{array}$ & Scotland & 165 & $\begin{array}{l}\text { Education through home visits and } \\
\text { telecare on knowledge and treat- } \\
\text { ment of HF. Educational brochure. } \\
\text { Instruments for self-monitoring. }\end{array}$ & 12 months & $\begin{array}{l}\text { Standard } \\
\text { care }\end{array}$ & $\begin{array}{l}\text { Readmissions* at } 12 \\
\text { months } \\
\text { Intervention: } 12 \\
\text { Control: } 26 \\
\text { Days of hospital stay+ } \\
\text { Intervention: } 3.43(12.2) \\
\text { Control: } 7.46(16.6)\end{array}$ \\
\hline $\begin{array}{l}\text { Boyde et } \\
\text { al., } 2018 \\
(13)\end{array}$ & $\begin{array}{l}\text { United } \\
\text { States }\end{array}$ & 200 & $\begin{array}{l}\text { Education prior to discharge on HF. } \\
\text { Brochure and video. }\end{array}$ & $\begin{array}{l}1,3 \text { and } 12 \\
\text { months }\end{array}$ & $\begin{array}{l}\text { Standard } \\
\text { care }\end{array}$ & $\begin{array}{l}\text { Readmissions* at } 3 \text { months } \\
\text { Intervention: } 8 \\
\text { Control: } 10 \\
\text { Readmissions* at } 12 \\
\text { months } \\
\text { Intervention: } 8 \\
\text { Control: } 14\end{array}$ \\
\hline $\begin{array}{l}\text { Brian et } \\
\text { al., } 2009 \\
(14)\end{array}$ & $\begin{array}{l}\text { United } \\
\text { States }\end{array}$ & 749 & $\begin{array}{l}\text { Education on HF. Brochure and } \\
\text { telephone follow-up. }\end{array}$ & 1 month & $\begin{array}{l}\text { Standard } \\
\text { care }\end{array}$ & $\begin{array}{l}\text { Readmissions* at } 1 \text { month } \\
\text { Intervention: } 55 \\
\text { Control: } 76\end{array}$ \\
\hline $\begin{array}{l}\text { Brotons et } \\
\text { al., } 2009 \\
(15)\end{array}$ & Spain & 283 & $\begin{array}{l}\text { Pre-discharge education on HF } \\
\text { with brochure. Home visits for one } \\
\text { year. Phone follow-up every } 15 \\
\text { days. }\end{array}$ & 12 months & $\begin{array}{l}\text { Standard } \\
\text { care }\end{array}$ & $\begin{array}{l}\text { Readmissions* at } 12 \\
\text { months } \\
\text { Intervention: } 52 \\
\text { Control: } 62\end{array}$ \\
\hline $\begin{array}{l}\text { Cañon- } \\
\text { Montañez } \\
\text { et al., } \\
2013 \\
(16)\end{array}$ & Colombia & 116 & $\begin{array}{l}\text { Education on } \mathrm{HF} \text { and its manage- } \\
\text { ment. Face to face and phone } \\
\text { education. }\end{array}$ & $\begin{array}{l}1 \text { and } 2 \\
\text { months }\end{array}$ & $\begin{array}{l}\text { Standard } \\
\text { care } \\
\text { (phone } \\
\text { call) }\end{array}$ & $\begin{array}{l}\text { Readmissions* at } 2 \text { months } \\
\text { Intervention: } 11 \\
\text { Control: } 5 \\
\text { Days of hospital stay at } 2 \\
\text { months } \\
\text { Intervention: } 6.27 \text { (5.93) } \\
\text { Control: } 11(11)\end{array}$ \\
\hline
\end{tabular}


Table 1. Characteristics of the included studies (Cont)

\begin{tabular}{|c|c|c|c|c|c|c|}
\hline $\begin{array}{l}\text { First } \\
\text { author, } \\
\text { year }\end{array}$ & Country & $\begin{array}{l}\text { Sam- } \\
\text { ple } \\
\text { size }\end{array}$ & Intervention group & Follow-up & $\begin{array}{l}\text { Control } \\
\text { group }\end{array}$ & Main outcomes \\
\hline $\begin{array}{l}\text { Cui et al., } \\
2019 \\
(17)\end{array}$ & China & 96 & $\begin{array}{l}\text { Structured education in HF for one } \\
\text { hour upon admission, and one } \\
\text { hour before discharge. Phone or } \\
\text { face-to-face consultation every } 4 \\
\text { weeks. }\end{array}$ & 12 months & $\begin{array}{l}\text { Standard } \\
\text { care }\end{array}$ & $\begin{array}{l}\text { Readmissions* at } 12 \\
\text { months } \\
\text { Intervention: } 5 \\
\text { Control: } 13\end{array}$ \\
\hline $\begin{array}{l}\text { Davis et } \\
\text { al., } 2012 \\
(18)\end{array}$ & $\begin{array}{l}\text { United } \\
\text { States }\end{array}$ & 125 & $\begin{array}{l}\text { Education during hospitalization. } \\
\text { Phone call after discharge. Video } \\
\text { with recorded sessions. Supplies to } \\
\text { aid self-care. }\end{array}$ & 1 month & $\begin{array}{l}\text { Standard } \\
\text { care }\end{array}$ & $\begin{array}{l}\text { Readmissions* at } 1 \text { month } \\
\text { Intervention: } 14 \\
\text { Control: } 12\end{array}$ \\
\hline $\begin{array}{l}\text { De Souza et } \\
\text { al., } 2014 \\
(19)\end{array}$ & Brazil & 252 & $\begin{array}{l}\text { Home visits to educate on HF. } \\
\text { Phone calls to reinforce education. }\end{array}$ & 6 months & $\begin{array}{l}\text { Standard } \\
\text { care }\end{array}$ & $\begin{array}{l}\text { Readmissions* at } 6 \text { months } \\
\text { Intervention: } 20 \\
\text { Control: } 30\end{array}$ \\
\hline $\begin{array}{l}\text { DeBusk et } \\
\text { al., } 2004 \\
(20)\end{array}$ & $\begin{array}{l}\text { United } \\
\text { States }\end{array}$ & 462 & $\begin{array}{l}\text { Education with a videotape. } \\
\text { Telephone counseling and printed } \\
\text { educational materials. }\end{array}$ & 12 months & $\begin{array}{l}\text { Standard } \\
\text { care }\end{array}$ & $\begin{array}{l}\text { Readmissions* at } 12 \\
\text { months } \\
\text { Intervention: } 76 \\
\text { Control: } 86\end{array}$ \\
\hline $\begin{array}{l}\text { Delaney et } \\
\text { al., } 2013 \\
(21)\end{array}$ & $\begin{array}{l}\text { United } \\
\text { States }\end{array}$ & 100 & $\begin{array}{l}\text { Telemonitoring. Brochure with } \\
\text { information on HF and its manage- } \\
\text { ment. }\end{array}$ & 3 months & $\begin{array}{l}\text { Standard } \\
\text { care }\end{array}$ & $\begin{array}{l}\text { Readmissions* at } 3 \text { months } \\
\text { Intervention: } 3 \\
\text { Control: } 7\end{array}$ \\
\hline $\begin{array}{l}\text { Dewalt et } \\
\text { al., } 2006 \\
(22)\end{array}$ & $\begin{array}{l}\text { United } \\
\text { States }\end{array}$ & 127 & $\begin{array}{l}\text { Education on HF and warning } \\
\text { signs. Phone calls for reinforce- } \\
\text { ment of the education. Educational } \\
\text { brochure. }\end{array}$ & $\begin{array}{l}6 \text { and } 12 \\
\text { months }\end{array}$ & $\begin{array}{l}\text { Standard } \\
\text { care }\end{array}$ & $\begin{array}{l}\text { Readmissions* at } 12 \\
\text { months } \\
\text { Intervention: } 18 \\
\text { Control: } 20\end{array}$ \\
\hline $\begin{array}{l}\text { Domingues } \\
\text { et al., } 2011 \\
(23)\end{array}$ & Brazil & 120 & $\begin{array}{l}\text { Phone calls after hospital dis- } \\
\text { charge to educate and evaluate } \\
\text { signs of decompensation of HF. }\end{array}$ & 3 months & $\begin{array}{l}\text { Standard } \\
\text { care }\end{array}$ & $\begin{array}{l}\text { Readmissions* at } 3 \text { months } \\
\text { Intervention: } 20 \\
\text { Control: } 23 \\
\text { Readmissions* at } 12 \\
\text { months } \\
\text { Intervention: } 22 \\
\text { Control: } 42 \\
\text { Days of hospital stay at } 12 \\
\text { months+ } \\
\text { Intervention: } 4.1(6.4) \\
\text { Control: } 7.6(12.1)\end{array}$ \\
\hline $\begin{array}{l}\text { Doughty et } \\
\text { al., } 2002 \\
(24)\end{array}$ & $\begin{array}{l}\text { New } \\
\text { Zealand }\end{array}$ & 197 & $\begin{array}{l}\text { Educational brochure on } \mathrm{HF} \text { and } \\
\text { its management. Home visits. }\end{array}$ & 12 months & $\begin{array}{l}\text { Standard } \\
\text { care }\end{array}$ & $\begin{array}{l}\text { Readmissions* at } 12 \\
\text { months } \\
\text { Intervention: } 36 \\
\text { Control: } 65\end{array}$ \\
\hline $\begin{array}{l}\text { Dracup et } \\
\text { al., } 2014 \\
(25)\end{array}$ & $\begin{array}{l}\text { United } \\
\text { States }\end{array}$ & 614 & $\begin{array}{l}\text { Face-to-face education session } \\
\text { delivered by a nurse focusing on } \\
\text { self-care. Phone calls. }\end{array}$ & $\begin{array}{l}3,12 \text { and } \\
24 \text { months }\end{array}$ & $\begin{array}{l}\text { Standard } \\
\text { care }\end{array}$ & $\begin{array}{l}\text { Readmissions* at } 24 \\
\text { months } \\
\text { Intervention: } 63 \\
\text { Control: } 64\end{array}$ \\
\hline $\begin{array}{l}\text { Ducharme } \\
\text { et al., } 2005 \\
(26)\end{array}$ & Canada & 230 & $\begin{array}{l}\text { Visits to the HF clinic to provide } \\
\text { education in the management of } \\
\text { the disease. Phone calls every } \\
\text { month. Educational brochure. }\end{array}$ & 6 months & $\begin{array}{l}\text { Standard } \\
\text { care }\end{array}$ & $\begin{array}{l}\text { Readmissions* at } 6 \text { months } \\
\text { Intervention: } 45 \\
\text { Control: } 66\end{array}$ \\
\hline
\end{tabular}


Table 1. Characteristics of the included studies (Cont)

\begin{tabular}{|c|c|c|c|c|c|c|}
\hline $\begin{array}{l}\text { First } \\
\text { author, } \\
\text { year }\end{array}$ & Country & $\begin{array}{l}\text { Sam- } \\
\text { ple } \\
\text { size }\end{array}$ & Intervention group & Follow-up & $\begin{array}{l}\text { Control } \\
\text { group }\end{array}$ & Main outcomes \\
\hline $\begin{array}{l}\text { Gámez- } \\
\text { López et } \\
\text { al., } 2012 \\
(27)\end{array}$ & Spain & 208 & $\begin{array}{l}\text { Follow-up in the HF clinic after } \\
\text { discharge. Phone call after dis- } \\
\text { charge to reinforce education. } \\
\text { Home visit. }\end{array}$ & 12 months & $\begin{array}{l}\text { Standard } \\
\text { care }\end{array}$ & $\begin{array}{l}\text { Readmissions* at } 12 \\
\text { months } \\
\text { Intervention: } 11 \\
\text { Control: } 14 \\
\text { Days of hospital stay at } 12 \\
\text { months+ } \\
\text { Intervention: } 6.7(13.5) \\
\text { Control: } 10.7(22.2)\end{array}$ \\
\hline $\begin{array}{l}\text { González- } \\
\text { Guerrero et } \\
\text { al., } 2014 \\
(28)\end{array}$ & Spain & 116 & $\begin{array}{l}\text { Flyer with information about the } \\
\text { disease. Follow-up call within } 48 \\
\text { hours. Reinforcement of education } \\
\text { after } 10 \text { days. Visits to the geriatric } \\
\text { center to reinforce education. }\end{array}$ & 12 months & $\begin{array}{l}\text { Standard } \\
\text { care }\end{array}$ & $\begin{array}{l}\text { Readmissions* at } 12 \\
\text { months } \\
\text { Intervention: } 14 \\
\text { Control: } 18 \\
\text { Days of hospital stay at } 12 \\
\text { months+ } \\
\text { Intervention: } 16.8(18.2) \\
\text { Control: } 20.6(23.5)\end{array}$ \\
\hline $\begin{array}{l}\text { Hägglund et } \\
\text { al., } 2015 \\
\text { (29) }\end{array}$ & Sweden & 72 & $\begin{array}{l}\text { Educational sessions at home } \\
\text { through a tablet about HF and its } \\
\text { management. }\end{array}$ & 3 months & $\begin{array}{l}\text { Standard } \\
\text { care }\end{array}$ & $\begin{array}{l}\text { Readmissions* at } 3 \text { months } \\
\text { Intervention: } 7 \\
\text { Control: } 11\end{array}$ \\
\hline $\begin{array}{l}\text { Holland et } \\
\text { al., } 2007 \\
(30)\end{array}$ & $\begin{array}{l}\text { United } \\
\text { Kingdom }\end{array}$ & 399 & $\begin{array}{l}\text { Home visit after discharge to } \\
\text { educate on HF and its manage- } \\
\text { ment. Follow-up visit to reinforce } \\
\text { education. }\end{array}$ & $\begin{array}{l}3 \text { and } 6 \\
\text { months }\end{array}$ & $\begin{array}{l}\text { Standard } \\
\text { care }\end{array}$ & $\begin{array}{l}\text { Readmissions* at } 3 \text { months } \\
\text { Intervention: } 12 \\
\text { Control: } 9 \\
\text { Readmissions* at } 6 \text { months } \\
\text { Intervention: } 1 \\
\text { Control: } 1\end{array}$ \\
\hline
\end{tabular}

$\begin{array}{llll}\begin{array}{l}\text { Jaarsma et } \\ \text { al., } 1999\end{array} & \begin{array}{l}\text { Nether- } \\ \text { (31) }\end{array} & 174 & \begin{array}{l}\text { Education about HF, treatment and } \\ \text { management during hospitaliza- } \\ \text { tion. Phone call and home visit. }\end{array}\end{array}$

1,3 and 9 Standard months care

Readmissions* at 3 months Intervention: 18

Control: 23

Days of hospital stay at 3 months +

Intervention: 3 (7)

Control: 4.1 (10)

$\begin{array}{ll}\text { Jaarsma et } & \text { Nether- } \\ \text { al., } 2011 & \text { lands }\end{array}$

1049 Home visit after discharge and every 6 months to receive education on HF. Additional home visits (basic group). Monthly contact with the nurse, additional visits and telephone follow-up (intensive group).

\section{Jerant et United al., $2001 \quad$ States} (33)

Kato et al., Japan 2016

(34)
Two home visits after discharge. Phone calls. Telecare.
Education and advice on knowledge about HF and self-care.
18 months $\begin{aligned} & \text { Standard } \\ & \text { care }\end{aligned}$

Readmissions* at 18

months

Intervention: 134

Control: 120

Standard
care

Readmissions* at 6 months Intervention: 1

Control: 4

24 months

Standard care
Readmissions* at 24

months

Intervention: 2

Control: 7 
Table 1. Characteristics of the included studies (Cont)

\begin{tabular}{|c|c|c|c|c|c|c|}
\hline $\begin{array}{l}\text { First } \\
\text { author, } \\
\text { year }\end{array}$ & Country & $\begin{array}{l}\text { Sam- } \\
\text { ple } \\
\text { size }\end{array}$ & Intervention group & Follow-up & $\begin{array}{l}\text { Control } \\
\text { group }\end{array}$ & Main outcomes \\
\hline $\begin{array}{l}\text { Kimmelstiel } \\
\text { et al., } 2004 \\
\text { (35) }\end{array}$ & $\begin{array}{l}\text { United } \\
\text { States }\end{array}$ & 200 & $\begin{array}{l}\text { Home visit. Manual with informa- } \\
\text { tion on HF. }\end{array}$ & $\begin{array}{l}3 \text { and } 6 \\
\text { months }\end{array}$ & $\begin{array}{l}\text { Standard } \\
\text { care }\end{array}$ & $\begin{array}{l}\text { Readmissions* at } 3 \text { months } \\
\text { Intervention: } 15 \\
\text { Control: } 24 \\
\text { Days of hospital stay at } \\
\text { months+ } \\
\text { Intervention: } 4.3(10.2) \\
\text { Control: } 7.8(19.7)\end{array}$ \\
\hline $\begin{array}{l}\text { Koelling et } \\
\text { al., } 2005 \\
(36)\end{array}$ & $\begin{array}{l}\text { United } \\
\text { States }\end{array}$ & 223 & $\begin{array}{l}\text { Education prior to discharge on the } \\
\text { management of HF. Information } \\
\text { brochure. Application of self-care } \\
\text { questionnaires. }\end{array}$ & $\begin{array}{l}1,3 \text { and } 6 \\
\text { months }\end{array}$ & $\begin{array}{l}\text { Standard } \\
\text { care }\end{array}$ & $\begin{array}{l}\text { Readmissions* at } 6 \text { months } \\
\text { Intervention: } 16 \\
\text { Control: } 33 \\
\text { Days of hospital stay at } 6 \\
\text { months+ } \\
\text { Intervention: } 13.1(36) \\
\text { Control: } 17.1 \text { (37) }\end{array}$ \\
\hline $\begin{array}{l}\text { Krumholz } \\
\text { et al., } \\
2002 \\
(37)\end{array}$ & $\begin{array}{l}\text { United } \\
\text { States }\end{array}$ & 88 & $\begin{array}{l}\text { Sequential education on HF and } \\
\text { its management. Educational bro- } \\
\text { chure. Home visits. Telemonitoring } \\
\text { to reinforce education. }\end{array}$ & 12 months & $\begin{array}{l}\text { Standard } \\
\text { care }\end{array}$ & $\begin{array}{l}\text { Readmissions* at } 12 \\
\text { months } \\
\text { Intervention: } 22 \\
\text { Control: } 42 \\
\text { Days of hospital stay at } 12 \\
\text { months+ } \\
\text { Intervention: } 4.1(6.4) \\
\text { Control: } 7.6(12.1)\end{array}$ \\
\hline $\begin{array}{l}\text { Leventhal et } \\
\text { al., } 2011 \\
\text { (38) }\end{array}$ & $\begin{array}{l}\text { Switzer- } \\
\text { land }\end{array}$ & 42 & $\begin{array}{l}\text { Home visit to provide HF educa- } \\
\text { tion. Phone calls to reinforce } \\
\text { education. Educational kit with } \\
\text { self-care procedures. }\end{array}$ & $\begin{array}{l}3,6,9 \text { and } \\
12 \text { months }\end{array}$ & $\begin{array}{l}\text { Standard } \\
\text { care }\end{array}$ & $\begin{array}{l}\text { Readmissions* at } 12 \\
\text { months } \\
\text { Intervention: } 1 \\
\text { Control: } 2\end{array}$ \\
\hline $\begin{array}{l}\text { Mau et al., } \\
2017 \\
\text { (39) }\end{array}$ & $\begin{array}{l}\text { United } \\
\text { States }\end{array}$ & 150 & $\begin{array}{l}\text { Educational modules on } \mathrm{HF} \text { and its } \\
\text { treatment. }\end{array}$ & 12 months & $\begin{array}{l}\text { Standard } \\
\text { care }\end{array}$ & $\begin{array}{l}\text { Readmissions* at } 12 \\
\text { months } \\
\text { Intervention: } 5 \\
\text { Control: } 18\end{array}$ \\
\hline $\begin{array}{l}\text { Melin et } \\
\text { al., } 2018 \\
(40)\end{array}$ & Sweden & 72 & $\begin{array}{l}\text { Education of self-care practices } \\
\text { and management of HF. }\end{array}$ & 6 months & $\begin{array}{l}\text { Standard } \\
\text { care }\end{array}$ & $\begin{array}{l}\text { Readmissions* at } 6 \text { months } \\
\text { Intervention: } 14 \\
\text { Control: } 16\end{array}$ \\
\hline $\begin{array}{l}\text { Naylor et } \\
\text { al., } 2004 \\
(41)\end{array}$ & $\begin{array}{l}\text { United } \\
\text { States }\end{array}$ & 239 & $\begin{array}{l}\text { Daily education during the hospi- } \\
\text { talization period. Home visits to } \\
\text { reinforce education about HF and } \\
\text { its management. }\end{array}$ & $\begin{array}{l}3,6 \text { and } 12 \\
\text { months }-2 \text {, } \\
6,12,26 \\
52 \text { weeks }\end{array}$ & $\begin{array}{l}\text { Standard } \\
\text { care }\end{array}$ & $\begin{array}{l}\text { Readmissions* at } 12 \\
\text { months } \\
\text { Intervention: } 40 \\
\text { Control: } 72 \\
\text { Days of hospital stay at } 12 \\
\text { months+ } \\
\text { Intervention: } 11.1(7.2) \\
\text { Control: } 14.5(13.4)\end{array}$ \\
\hline $\begin{array}{l}\text { Negarandeh } \\
\text { et al., } 2019 \\
\text { (42) }\end{array}$ & Iran & 80 & Telemonitoring with HF education. & $\begin{array}{l}1 \text { and } 3 \\
\text { months }\end{array}$ & $\begin{array}{l}\text { Standard } \\
\text { care }\end{array}$ & $\begin{array}{l}\text { Readmissions* at } 3 \text { months } \\
\text { Intervention: } 7 \\
\text { Control: } 14\end{array}$ \\
\hline $\begin{array}{l}\text { Otsu et al., } \\
2011 \\
(43)\end{array}$ & Japan & 102 & $\begin{array}{l}\text { Educational program in } \mathrm{HF} \text { clinic } \\
\text { about the disease and its manage- } \\
\text { ment. }\end{array}$ & $\begin{array}{l}3,6,9 \text { and } \\
12 \text { months }\end{array}$ & $\begin{array}{l}\text { Standard } \\
\text { care }\end{array}$ & $\begin{array}{l}\text { Readmissions* at } 6 \text { months } \\
\text { Intervention: } 1 \\
\text { Control: } 1\end{array}$ \\
\hline
\end{tabular}


Table 1. Characteristics of the included studies (Cont)

\begin{tabular}{|c|c|c|c|c|c|c|}
\hline $\begin{array}{l}\text { First } \\
\text { author, } \\
\text { year }\end{array}$ & Country & $\begin{array}{l}\text { Sam- } \\
\text { ple } \\
\text { size }\end{array}$ & Intervention group & Follow-up & $\begin{array}{l}\text { Control } \\
\text { group }\end{array}$ & Main outcomes \\
\hline $\begin{array}{l}\text { Ramachan- } \\
\text { dran et al., } \\
2007 \\
(44)\end{array}$ & India & 50 & $\begin{array}{l}\text { Education on HF, management } \\
\text { and treatment. Reinforcement of } \\
\text { education by phone calls. Patient } \\
\text { education manual. Follow-up in } \\
\text { the HF clinic. }\end{array}$ & 6 months & $\begin{array}{l}\text { Standard } \\
\text { care }\end{array}$ & $\begin{array}{l}\text { Readmissions* at } 6 \text { months } \\
\text { Intervention: } 6 \\
\text { Control: } 4\end{array}$ \\
\hline $\begin{array}{l}\text { Rodríguez- } \\
\text { Gázquez et } \\
\text { al., } 2012 \\
(45)\end{array}$ & Colombia & 63 & $\begin{array}{l}\text { Educational program in nursing } \\
\text { (educational meetings, home vis- } \\
\text { its, telenursing and a printed book) } \\
\text { in the improvement of self-care } \\
\text { behaviors. }\end{array}$ & 9 months & $\begin{array}{l}\text { Standard } \\
\text { care }\end{array}$ & $\begin{array}{l}\text { Readmissions* at } 9 \text { months } \\
\text { Intervention: } 30 \\
\text { Control: } 24\end{array}$ \\
\hline $\begin{array}{l}\text { Ruschel et } \\
\text { al., } 2018 \\
(46)\end{array}$ & Brazil & 252 & $\begin{array}{l}\text { Home visits and phone calls. } \\
\text { Education on HF and self-care } \\
\text { practices. }\end{array}$ & 6 months & $\begin{array}{l}\text { Standard } \\
\text { care }\end{array}$ & $\begin{array}{l}\text { Readmissions* at } 6 \text { months } \\
\text { Intervention: } 30 \\
\text { Control: } 30\end{array}$ \\
\hline $\begin{array}{l}\text { Sethares et } \\
\text { al., } 2004 \\
(47)\end{array}$ & $\begin{array}{l}\text { United } \\
\text { States }\end{array}$ & 70 & $\begin{array}{l}\text { Education about HF during hospi- } \\
\text { talization. Reinforcement education } \\
\text { after discharge. }\end{array}$ & 3 months & $\begin{array}{l}\text { Standard } \\
\text { care }\end{array}$ & $\begin{array}{l}\text { Readmissions* at } 3 \text { months } \\
\text { Intervention: } 6 \\
\text { Control: } 12\end{array}$ \\
\hline $\begin{array}{l}\text { Stewart et } \\
\text { al., } 2015 \\
(48)\end{array}$ & $\begin{array}{l}\text { Australia } \\
\text { and } \\
\text { New } \\
\text { Zealand }\end{array}$ & 624 & $\begin{array}{l}\text { Home visit after discharge. Educa- } \\
\text { tion on HF and its management. } \\
\text { Personalized care plan. }\end{array}$ & $\begin{array}{l}1 \text { and } 36 \\
\text { months }\end{array}$ & $\begin{array}{l}\text { Standard } \\
\text { care }\end{array}$ & $\begin{array}{l}\text { Readmissions* at } 36 \\
\text { months } \\
\text { Intervention: } 17 \\
\text { Control: } 17\end{array}$ \\
\hline $\begin{array}{l}\text { Tomita et } \\
\text { al., } 2009 \\
(49)\end{array}$ & $\begin{array}{l}\text { United } \\
\text { States }\end{array}$ & 40 & $\begin{array}{l}\text { Information online about HF and } \\
\text { its management. }\end{array}$ & $\begin{array}{l}6 \text { and } 12 \\
\text { months }\end{array}$ & $\begin{array}{l}\text { Standard } \\
\text { care }\end{array}$ & $\begin{array}{l}\text { Days of hospital stay at } 6 \\
\text { months+ } \\
\text { Intervention: } 1(2.45) \\
\text { Control: } 0.84(1.89) \\
\text { Days of hospital stay at } 12 \\
\text { months+ } \\
\text { Intervention: } 1.23(2.55) \\
\text { Control: } 2.42(5.07)\end{array}$ \\
\hline $\begin{array}{l}\text { Tsuchi- } \\
\text { hashi- } \\
\text { Makaya et } \\
\text { al., } 2013 \\
\text { (50) }\end{array}$ & Japan & 164 & $\begin{array}{l}\text { Pre-discharge education on HF } \\
\text { and its management. Educational } \\
\text { brochure. Home visits once a week } \\
\text { for two months. Monthly telephone } \\
\text { follow-up for six months. }\end{array}$ & $\begin{array}{l}2,6 \text { and } 12 \\
\text { months }\end{array}$ & $\begin{array}{l}\text { Standard } \\
\text { care }\end{array}$ & $\begin{array}{l}\text { Readmissions* at } 6 \text { months } \\
\text { Intervention: } 6 \\
\text { Control: } 15 \\
\text { Readmissions* at } 12 \\
\text { months } \\
\text { Intervention: } 6 \\
\text { Control: } 9\end{array}$ \\
\hline $\begin{array}{l}\text { Wakefield } \\
\text { et al., } \\
2008 \\
(51)\end{array}$ & $\begin{array}{l}\text { United } \\
\text { States }\end{array}$ & 148 & $\begin{array}{l}\text { Follow-up after discharge. Phone } \\
\text { calls to provide HF education. }\end{array}$ & $\begin{array}{l}3,6 \text { and } 12 \\
\text { months }\end{array}$ & $\begin{array}{l}\text { Standard } \\
\text { care }\end{array}$ & $\begin{array}{l}\text { Readmissions* at } 12 \\
\text { months } \\
\text { Intervention: } 21 \\
\text { Control: } 29\end{array}$ \\
\hline $\begin{array}{l}\text { Wierzchow- } \\
\text { iecki et al., } \\
2006 \\
\text { (52) }\end{array}$ & Poland & 160 & $\begin{array}{l}\text { Education and follow-up in the HF } \\
\text { clinic. Phone calls for educational } \\
\text { reinforcement. }\end{array}$ & 12 months & $\begin{array}{l}\text { Standard } \\
\text { care }\end{array}$ & $\begin{array}{l}\text { Readmissions* at } 12 \\
\text { months } \\
\text { Intervention: } 13 \\
\text { Control: } 25\end{array}$ \\
\hline
\end{tabular}


Table 1. Characteristics of the included studies (Cont)

\begin{tabular}{|c|c|c|c|c|c|c|}
\hline $\begin{array}{l}\text { First } \\
\text { author, } \\
\text { year }\end{array}$ & Country & $\begin{array}{l}\text { Sam- } \\
\text { ple } \\
\text { size }\end{array}$ & Intervention group & Follow-up & $\begin{array}{l}\text { Control } \\
\text { group }\end{array}$ & Main outcomes \\
\hline $\begin{array}{l}\text { Wright et } \\
\text { al., } 2003 \\
\text { (53) }\end{array}$ & $\begin{array}{l}\text { New } \\
\text { Zealand }\end{array}$ & 197 & $\begin{array}{l}\text { Clinical review after discharge. } \\
\text { Home visits every } 6 \text { weeks to } \\
\text { educate on } \mathrm{HF} \text {, treatment and } \\
\text { management. }\end{array}$ & 12 months & $\begin{array}{l}\text { Standard } \\
\text { care }\end{array}$ & $\begin{array}{l}\text { Readmissions* at } 12 \\
\text { months } \\
\text { Intervention: } 46 \\
\text { Control: } 18 \\
\text { Days of hospital stay at } 12 \\
\text { months+ } \\
\text { Intervention: } 9.4(13.6) \\
\text { Control: } 14.9 \text { (18.8) }\end{array}$ \\
\hline $\begin{array}{l}\text { Yu et al., } \\
2015 \\
(54)\end{array}$ & China & 178 & $\begin{array}{l}\text { Education before discharge about } \\
\text { HF. Home visits and phone calls } \\
\text { for educational reinforcement. }\end{array}$ & $\begin{array}{l}6 \text { weeks, } \\
3 \text { and } 9 \\
\text { months }\end{array}$ & $\begin{array}{l}\text { Standard } \\
\text { care }\end{array}$ & $\begin{array}{l}\text { Readmissions* at } 6 \text { weeks - } \\
3 \text { months }-9 \text { months } \\
\text { Intervention: } 7-12-6 \\
\text { Control: } 10-7-3\end{array}$ \\
\hline
\end{tabular}

HF: heart failure; * Data presented as number of patients readmitted due to decompensation of HF; + Data presented as mean (standard deviation).

Table 1 shows that this SR included 9688 adult patients with HF. The studies were published between 1999 and 2019. The investigations were conducted in 16 countries, with the highest number of these in the United States and Spain (16 and 5, respectively). The follow-up of the studies included was carried out during different periods, comprised between the first month after the intervention and at 36 months. Studies with follow-up at 3,6 and 12 months were predominant.

With respect to the educational interventions, these were diverse; however, common strategies were found in the studies included, like: education during hospitalization, telephone follow-up, home visits to reinforce the education, visits to HF clinics, and delivery of printed or digital educational material (brochures, videos or manuals) for consultation by the patients. The education centered on knowledge of the disease, warning signs, diet, and self-care practices.

Regarding the comparison with the control group, it was found that in general, the usual care was perceived as the clinical care by the cardiologist and a single control visit in the outpatient care service.

\section{Analysis of the risk of bias of the studies included}

The evaluation of the risk of bias of the studies is presented in Table 2. According with the parameters evaluated by the RoB 1 tool, ${ }^{(9)}$ it was obtained that all the studies performed an adequate random sequence generation; allocation concealment was optimal in $65.1 \%$ of the studies included. Due to the nature of the educational interventions, in the studies it was not possible to conduct blinding of the patients and of the staff who offered the interventions. In relation blinding of outcome assessment, only $48.8 \%$ low risk was presented for this domain. In all, $93 \%$ of the studies described clearly the losses presented during the follow-up and if the data analysis was carried out through intention of treatment, which reduced the risk of bias due to incomplete results. Finally, regarding the risk of selective reporting of the results, it was found that $97.7 \%$ described the results proposed since the beginning (Table 2). 


\section{Table 2. Assessment of risk of bias among included studies}

\begin{tabular}{|c|c|c|c|c|c|c|}
\hline Studies & $\begin{array}{l}\text { Random } \\
\text { sequence } \\
\text { generation }\end{array}$ & $\begin{array}{l}\text { Allocation } \\
\text { concealment }\end{array}$ & $\begin{array}{l}\text { Blinding of } \\
\text { participants } \\
\text { and personnel }\end{array}$ & $\begin{array}{l}\text { Blinding of } \\
\text { outcome as- } \\
\text { sessment }\end{array}$ & $\begin{array}{l}\text { Incomplete } \\
\text { outcome } \\
\text { data }\end{array}$ & $\begin{array}{l}\text { Selective } \\
\text { reporting }\end{array}$ \\
\hline Aldamiz-Echevarría et al., 2007 (10) & Low risk & Low risk & Low risk & Low risk & Low risk & Low risk \\
\hline Atienza et al., 2004 (11) & Low risk & Low risk & Low risk & Unclear risk & Low risk & Low risk \\
\hline Blue et al., 2001 (12) & Low risk & Low risk & Low risk & Unclear risk & Low risk & Low risk \\
\hline Boyde et al., 2018 (13) & Low risk & Low risk & Low risk & High risk & Low risk & Low risk \\
\hline Brian et al., 2009 (14) & Low risk & Low risk & Low risk & Low risk & Low risk & Low risk \\
\hline Brotons et al., 2009 (15) & Low risk & Low risk & Low risk & Low risk & Low risk & Low risk \\
\hline Cañon-Montañez et al., 2013 (16) & Low risk & Low risk & Low risk & Low risk & Low risk & Low risk \\
\hline Cui et al., 2019 (17) & Low risk & Low risk & Low risk & Low risk & Low risk & Low risk \\
\hline Davis et al., 2012 (18) & Low risk & Unclear risk & Low risk & Low risk & Low risk & Low risk \\
\hline De Souza et al., 2014 (19) & Low risk & Low risk & Low risk & Low risk & Low risk & Low risk \\
\hline DeBusk et al., 2004 (20) & Low risk & Low risk & Low risk & Low risk & Low risk & Low risk \\
\hline Delaney et al., 2013 (21) & Low risk & Low risk & Low risk & Unclear risk & Low risk & Low risk \\
\hline Dewalt et al., 2006 (22) & Low risk & Low risk & Low risk & High risk & Low risk & Low risk \\
\hline Domingues et al., 2011 (23) & Low risk & Unclear risk & Low risk & Unclear risk & Unclear risk & Low risk \\
\hline Doughty et al., 2002 (24) & Low risk & Low risk & Low risk & Low risk & Low risk & Low risk \\
\hline Dracup et al., 2014 (25) & Low risk & Low risk & Low risk & Low risk & Low risk & Low risk \\
\hline Ducharme et al., 2005 (26) & Low risk & Low risk & Low risk & Low risk & Low risk & Low risk \\
\hline Gámez-López et al., 2012 (27) & Low risk & Unclear risk & Low risk & Low risk & Unclear risk & Low risk \\
\hline González-Guerrero et al., 2014 (28) & Low risk & Low risk & Low risk & Unclear risk & Low risk & Low risk \\
\hline Hägglund et al., 2015 (29) & Low risk & Unclear risk & Low risk & Unclear risk & Low risk & Low risk \\
\hline Holland et al., 2007 (30) & Low risk & Low risk & Low risk & Unclear risk & Low risk & Low risk \\
\hline Jaarsma et al., 1999 (31) & Low risk & Low risk & Low risk & Low risk & Low risk & Low risk \\
\hline Jaarsma et al., 2011 (32) & Low risk & Low risk & Low risk & Unclear risk & Low risk & Low risk \\
\hline Jerant et al., 2001(33) & Low risk & Low risk & Low risk & Unclear risk & Low risk & Low risk \\
\hline Kato et al., 2016 (34) & Low risk & Low risk & Low risk & Low risk & Low risk & Low risk \\
\hline Kimmelstiel et al., 2004 (35) & Low risk & High risk & Low risk & Low risk & Low risk & Low risk \\
\hline Koelling et al., 2005 (36) & Low risk & Low risk & Low risk & Low risk & Low risk & Low risk \\
\hline Krumholz et al., 2002 (37) & Low risk & Unclear risk & Low risk & Unclear risk & Low risk & Unclear risk \\
\hline Leventhal et al., 2011 (38) & Low risk & Low risk & Low risk & Low risk & Low risk & Low risk \\
\hline
\end{tabular}




\section{Table 2. Assessment of risk of bias among included studies (Cont)}

\begin{tabular}{|c|c|c|c|c|c|c|}
\hline Studies & $\begin{array}{l}\text { Random } \\
\text { sequence } \\
\text { generation }\end{array}$ & $\begin{array}{l}\text { Allocation } \\
\text { concealment }\end{array}$ & $\begin{array}{l}\text { Blinding of } \\
\text { participants } \\
\text { and personnel }\end{array}$ & $\begin{array}{l}\text { Blinding of } \\
\text { outcome as- } \\
\text { sessment }\end{array}$ & $\begin{array}{l}\text { Incomplete } \\
\text { outcome } \\
\text { data }\end{array}$ & $\begin{array}{l}\text { Selective } \\
\text { reporting }\end{array}$ \\
\hline Mau et al., 2017 (39) & Low risk & High risk & Low risk & Unclear risk & Low risk & Low risk \\
\hline Melin et al., 2018 (40) & Low risk & High risk & Low risk & Low risk & Low risk & Low risk \\
\hline Naylor et al., 2004 (41) & Low risk & Low risk & Low risk & Low risk & Low risk & Low risk \\
\hline Negarandeh et al., 2019 (42) & Low risk & Low risk & Low risk & Unclear risk & Low risk & Low risk \\
\hline Otsu et al., 2011 (43) & Low risk & Unclear risk & Low risk & High risk & Low risk & Low risk \\
\hline Ramachandran et al., 2007 (44) & Low risk & Low risk & Low risk & High risk & Low risk & Low risk \\
\hline Rodríguez-Gázquez et al., 2012 (45) & Low risk & Low risk & Low risk & Unclear risk & Low risk & Low risk \\
\hline Ruschel et al., 2018 (46) & Low risk & Unclear risk & Low risk & Low risk & Low risk & Low risk \\
\hline Sethares et al., 2004 (47) & Low risk & Low risk & Low risk & Low risk & Low risk & Low risk \\
\hline Stewart et al., 2015 (48) & Low risk & Low risk & Low risk & Low risk & Low risk & Low risk \\
\hline Tomita et al., 2009 (49) & Low risk & Unclear risk & Low risk & Unclear risk & Low risk & Low risk \\
\hline Tsuchihashi Makaya et al. 2013 (50) & Low risk & Unclear risk & Low risk & Unclear risk & Low risk & Low risk \\
\hline Walkefield et al., 2008 (51) & Low risk & Low risk & Low risk & Unclear risk & Low risk & Low risk \\
\hline Wierzchowiecki et al., 2006 (52) & Low risk & Unclear risk & Low risk & Unclear risk & Unclear risk & Low risk \\
\hline Wright et al., 2003 (53) & Low risk & Unclear risk & Low risk & Unclear risk & Low risk & Low risk \\
\hline Yu et al., 2015 (54) & Low risk & Low risk & Low risk & Unclear risk & Low risk & Low risk \\
\hline
\end{tabular}

\section{Meta-analysis}

The work included the results from 43 studies and analyzed hospital readmissions, during different follow-up periods, i.e., 6 weeks, 1 month, 2, 3, 6, 9, 12 , and 24 months. Upon evaluating the combined effect, no statistically significant results were obtained in studies with follow-up $<3$ months nor at three months (Figure 2). Significant results were also not found at nine months (RR: $0.98,95 \% \mathrm{Cl}$ : 0.64 to $\left.1.54,1^{2}: 61 \%\right)$, as well as at 24 months (RR: $0.72,95 \% \mathrm{Cl}: 0.24$ to $2.17, \mathrm{I}^{2}$ : $62 \%$ ). 


Events Total Events Tot

Boyde, 2018

Brian, 2009

Cañón-Montañez, 2013

Davis, 2012

Holland, 2007

Jaarsma, 1999

Tsuchihashi-Makaya, 2013

Yu, 2015

$\begin{array}{ll}7 & 98\end{array}$

$98 \quad 4$

97

M-H, Random, 95\% Cl

M-H, Random, $95 \% \mathrm{Cl}$

Total ( $95 \% \mathrm{Cl})$

$55 \quad 373$

$11 \quad 58$

$14 \quad 55$

$42 \quad 148$

$8 \quad 84$

$4 \quad 79$

$1.73[0.52,5.73]$

$0.73[0.53,1.00]$

$2.20[0.82,5.94]$

$1.15[0.58,2.25]$

$0.83[0.59,1.17]$

$0.82[0.35,1.95]$

$1.04[0.27,4.01]$

$0.68[0.27,1.72]$

Total events

985

Heterogeneity: $\mathrm{Tau}^{2}=0.00 ; \mathrm{Chi}^{2}=6.87, \mathrm{df}=7(\mathrm{P}=0.44) ; \mathrm{P}^{2}=0 \%$

Test for overall effect: $\mathrm{Z}=1.54(\mathrm{P}=0.12)$

$0.86[0.70,1.04]$

$993 \quad 100.0 \%$

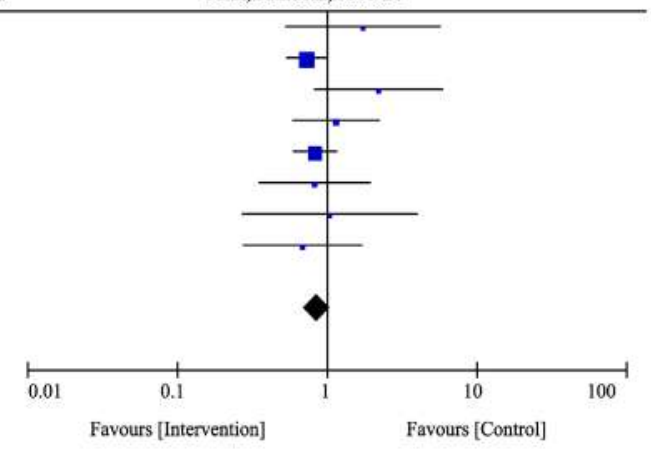

Intervention

Control

Risk Ratio

Risk Ratio

Study or Subgroup Events Total Events Total Weight $\mathrm{M}-\mathrm{H}$, Random, $\mathbf{9 5} \% \mathrm{Cl}$

M-H, Random, $95 \% \mathrm{Cl}$

Boyde, 2018

Delaney, 2013

Domingues, 2011

Hagglund, 2015

Holland, 2007

Jaarsma, 1999

Kimmelstiel, 2004

Negarandeh, 2019

Sethares, 2004

Yu, 2015

$\begin{array}{ll}8 & 92 \\ 3 & 46\end{array}$

46

94

$6.4 \%$ $0.82[0.34,1.98]$

$0.44[0.12,1.59]$

$1.14[0.72,1.82]$

$0.80[0.35,1.82]$

$\begin{array}{lllll}7 & 32 & 11 & 40 & 7.3 \%\end{array}$

$7.3 \%$

$1.29[0.56,2.96]$

$0.89[0.51,1.52]$

$0.66[0.37,1.19]$

$0.47[0.22,1.02]$

$0.56[0.24,1.33]$

$1.51[0.63,3.62]$

Total $(95 \% \mathrm{Cl})$

691

Total events

108

$8.4 \%$

$6.8 \%$

Heterogeneity: $\mathrm{Tau}^{2}=0.00 ; \mathrm{Chi}^{2}=9.07, \mathrm{df}=9(\mathrm{P}=0.43) ; \mathrm{F}^{2}=1 \%$

Test for overall effect: $\mathrm{Z}=1.42(\mathrm{P}=0.16)$

140

$722 \quad 100.0 \%$

$0.85[0.68,1.06]$

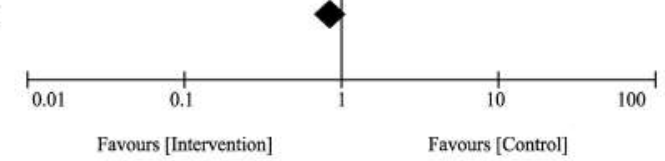

Figure 2. Meta-analysis of the effect of the educational

interventions on reducing readmissions due to heart failure. (A)

Follow-up $<3$ months, (B) Follow-up at 3 months

The MA of studies with follow-up at six months showed a $30 \%$ decrease in readmissions (RR: 0.70; 95\% Cl: 0.58 to $0.84 ; I^{2}$ : $0 \%$ ) and the 12 -month follow-up evidenced 33\% reduction (RR: 0.67; 95\% $\mathrm{Cl}: 0.58$ to 0.76 ; $\mathrm{I}^{2}$ : $52 \%$ ); both analyses in favor of the group of educational interventions (Figure 3 ). 
Intervention Control

Risk Ratio

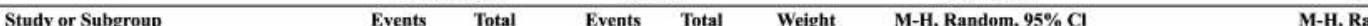

$\begin{array}{lllllll}\text { De Souza, } 2014 & 20 & 117 & 30 & 126 & 12.9 \% & 0.72[0.43,1.19]\end{array}$

Holland, 2007

Jerant, 2001

Koelling, 2005

Melin, 2018

Otsu, 2011

Ramachandran, 2007

Ruschel, 2018

Tsuchihashi-Makaya, 2013

Total $(95 \% \mathrm{Cl})$

Total events

$\begin{array}{rr}117 & 30 \\ 115 & 6 \\ 148 & \\ 13 & \\ 107 & 33 \\ 32 & 16 \\ 49 & \\ 25 & \\ 117 & 3 \\ 79 & 15\end{array}$

Heterogeneity: $\mathrm{Tau}^{2}=0.00 ; \mathrm{Chi}^{2}=8.03, \mathrm{df}=9(\mathrm{P}=0.53) ; 1^{2}=0 \%$

802

$\begin{array}{lll}126 & 12.9 \% & 0.72[0.43,1.19 \\ 115 & 43.2 \% & 0.68[0.52,0.90]\end{array}$

$0.97[0.06,15.30]$

$0.23[0.03,1.79]$

$0.53[0.31,0.90]$

$1.09[0.63,1.89]$

$0.96[0.06,14.90$

$1.50[0.48,4.68]$

$0.70[0.42,1.16$

Test for overall effect: $Z=3.83(P=0.0001)$

$0.70[0.58,0.84$

Aldamiz-Echevarria, 2007

Atienza, 2004

Blue, 2001

Boyde, 2018

Brotons, 2009

Cui, 2019

DeBusk, 2004

Dewalt, 2006

Domingues, 2011

Doughty, 2002

González-Guerrero, 2014

Gamez-López, 2012

Leventhal, 2011

Mau, 2017

Naylor, 2004

Tsuchihashi-Makaya, 2013

Wakefield, 2008

Wierzchowiecki, 2006

Wright, 2003

Krumholz, 2002

Events 55

Total $(95 \% \mathrm{CI})$

Total events

\begin{tabular}{rr} 
Total & Even \\
137 & \\
164 & 127 \\
84 & \\
83 & \\
144 & \\
48 & \\
199 & \\
52 & \\
44 & \\
100 & \\
59 & \\
52 & \\
22 & \\
57 & \\
118 & \\
79 & \\
33 & \\
80 & \\
76 & \\
44 & \\
\hline 5 &
\end{tabular}

$\begin{array}{rrrr}57 & 142 & 7.5 \% & \end{array}$

$1.00[0.75,1.33]$

$0.53[0.43,0.66]$

$0.45[0.24,0.82]$

$0.61[0.27,1.37]$

$0.81[0.61,1.08]$

$0.38[0.15,1.00]$

$0.85[0.67,1.07]$

$1.02[0.61,1.71]$

$0.52[0.39,0.71]$

$0.54[0.40,0.72]$

$0.76[0.42,1.39]$

$0.79[0.39,1.57]$

$0.45[0.04,4.64]$

$0.34[0.13,0.86]$

$0.57[0.43,0.76]$

$0.69[0.26,1.85]$

$0.92[0.66,1.28]$

$0.52[0.29,0.94$

$0.81[0.60,1.08]$

$0.52[0.39,0.71]$

1675

524

$00.0 \% \quad 0.67[0.58,0.76]$

Heterogeneity: $\mathrm{Tau}^{2}=0.04 ; \mathrm{Ch}^{2}=39.79, \mathrm{df}=19(\mathrm{P}=0.003) ; \mathrm{I}^{\mathrm{z}}=52 \%$

Test for overall effect: $\mathrm{Z}=3.83(\mathrm{P}=0.0001)$

$1656 \quad 100.0 \%$

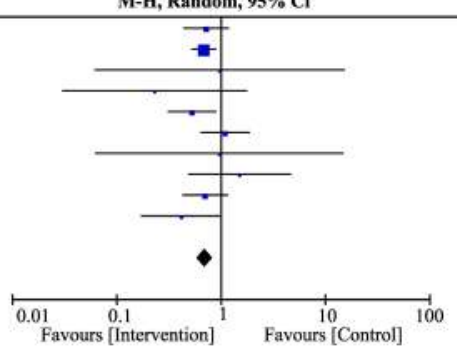

Figure 3. Meta-analysis of the effect of educational

interventions on reducing readmissions due to heart failure.

(A) Follow-up at 6 months, (B) Follow-up at 12 months 


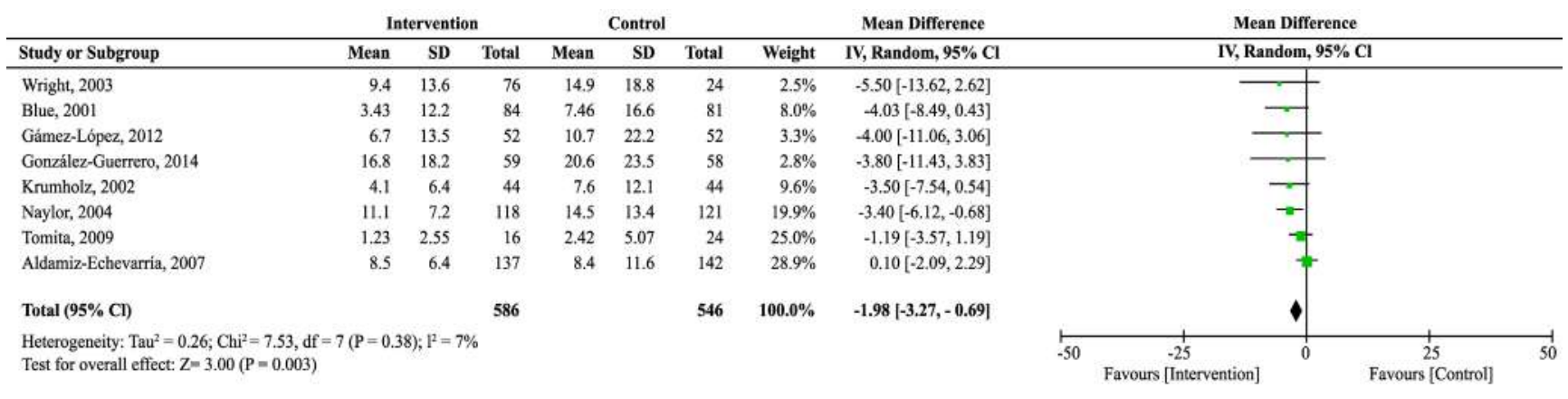

Figure 4. Meta-analysis of the effect of educational interventions on reducing days of hospital stay due to heart failure at 12 months of follow-up

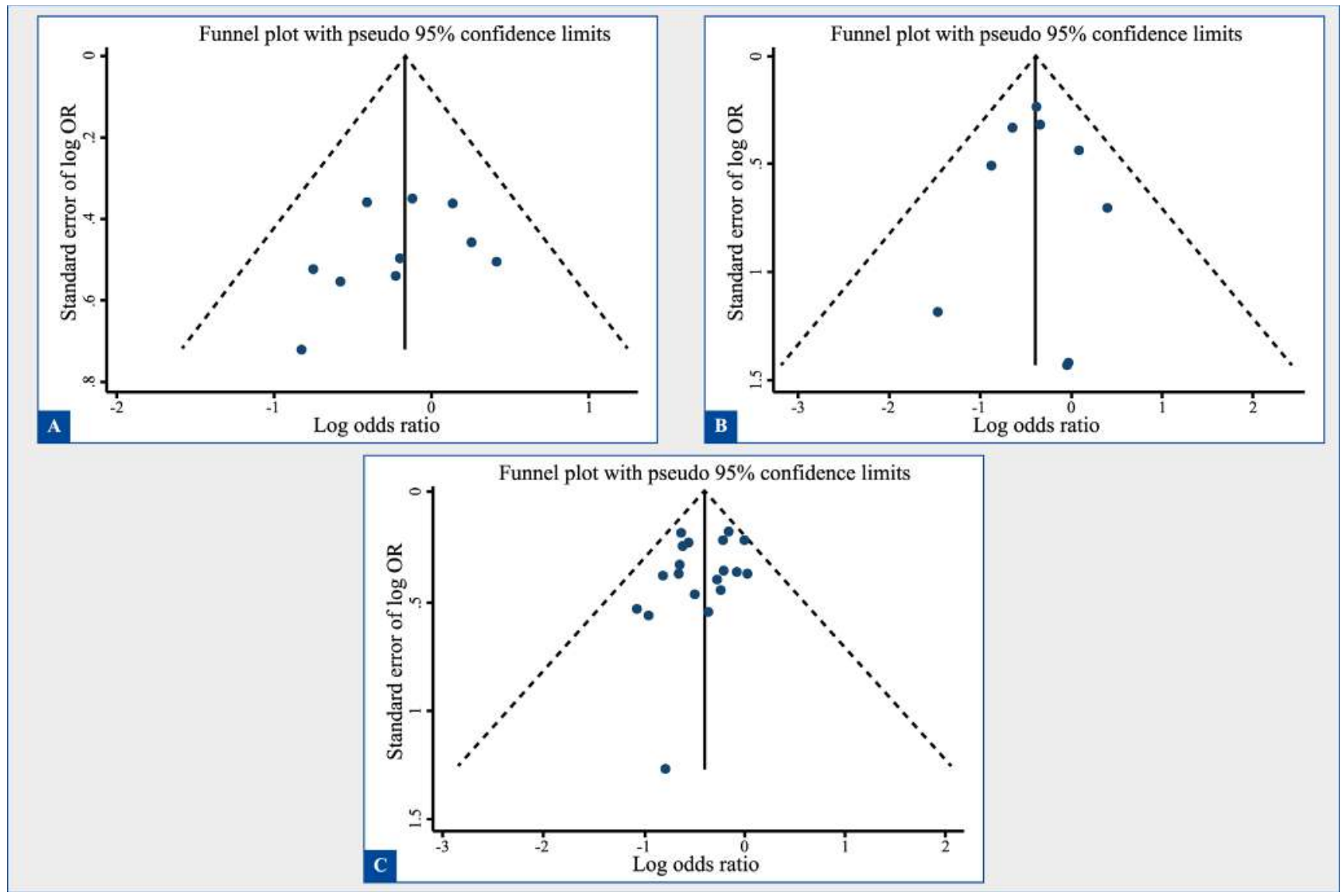

Figure 5. Funnel plot to analyze publication bias or bias due to missing results during three follow-up times. (A) 3 months, (B) 6 months, (C) 12 months 
For the secondary outcome, days of hospital stay, no favorable effect was found of the educational interventions during the follow-up at three months (MD: $-1.71 ; 95 \% \mathrm{Cl}:-3.87$ to $-0.46 ; \mathrm{I}^{2}$ : $0 \%$ ) and six months (MD: $0.07 ; 95 \% \mathrm{Cl}:-1.33$ to 1.45 ; $1^{2}$ : 0\%). Nevertheless, the MA with follow-up at 12 months (Figure 4) evidenced a reduction of approximately two days in patients who received the educational interventions (MD: $-1.98 ; 95 \%$ $\mathrm{Cl}:-3.27$ to $-0.69 ; \mathrm{I}^{2}$ : 7\%).

\section{Evaluation of publication bias or bias due to missing results}

Figure 5 shows funnel plot graphics to evaluate publication bias under analysis of 10 or more studies (three months, six months, and twelve months of follow-up). For the three times of follow-up, it is possible to observe generally a funnel shape that indicates that the studies are distributed uniformly on both sides of the average, which suggests lack of publication bias. The Egger statistical test also indicated absence of publication bias ( 3 months, $p=0.30$; 6 months, $p=0.87$, and 12 months, $p=0.26$ ).

\section{Discussion}

This up-to-date synthesis of the evidence shows the favorable combined effect of educational interventions during prolonged follow-up times (six and twelve months) to reduce readmissions and time of hospital stay in adults with HF. These results are coherent with other SR and MA conducted prior to this study. ${ }^{(55-57)}$ In addition, the results found reinforce the importance of education for patients and of the multidisciplinary management of the HF syndrome. Similarly, these educational strategies become an alternative of effective intervention to improve the clinical outcomes of patients and which can be useful to reduce costs associated with health services due to HF decompensation. Within this context, a 2017 SR ${ }^{(55)}$ concluded that educational interventions, especially those guided by nurses, have positive effects on decreasing readmissions due to HF.

Two of its studies, which are also part of this $\mathrm{SR}^{(38,42)}$ evidenced $50 \%$ reduction in readmissions when patients were subjected to educational interventions. In addition, an MA from 2019,(56) that included seven of the RCTs from this study, demonstrates a reduction in hospital readmissions due to HF in follow-up from 6 to 12 months of 27\% (RR: $0.73 ; 95 \% \mathrm{Cl}: 0.61$ to $0.88 ; 1^{2}$ : $0 \%$ ) and a general $22 \%$ reduction, which groups all the follow-up times. The previously stated, reaffirms the results obtained in this study and gives value to educational interventions as a low-cost strategy to improve the clinical response of patients with HF.

Likewise, another MA from 2019,(57) obtained similar data. The researchers showed reduction of readmissions at 12 months of $36 \%$ (RR: 0.64 ; 95\% Cl: 0.53 to $\left.0.78 ;\left.\right|^{2}: 51 \%\right)$. Moreover, this study also evidenced a decrease of approximately two days in hospital stay of adult patients with $\mathrm{HF}$ at 12-month follow-up and favorable for the educational interventions. However, no evidence was found of other SR or MA that have evaluated the effect of educational interventions for this result, becoming a significant contribution of this SR and which opens an important path to study this clinical outcome. ${ }^{(57)}$ These results of the evidence can be a starting point to restructure nursing care and management programs for adults with HF. A proactive scenario is proposed in which patients after their discharge continue being a priority and responsibility for health institutions to avoid new readmissions. The findings of studies with prolonged follow-up times show that companionship and active monitoring of patients by a multidisciplinary team generate a positive impact on the clinical outcomes of patients. ${ }^{(56,57)}$

Another relevant aspect of this $\mathrm{SR}$ is that the educational interventions from the studies selected were variables on frequency, duration, methodology and personnel in charge of conducting them. Nevertheless, it is worth highlighting that a vast number of them were carried out by the nursing 
staff experienced in the cardiovascular area, which reinforces the importance of the nurses' educator role as an effective strategy in reducing hospital readmissions and maintaining the quality of life of patients with HF. The aforementioned is based on nurses being the professionals called on to provide primary care in patients with chronic diseases. ${ }^{(58,59)}$

Also, it is important to mention although the study followed the methodological recommendations by the Cochrane Collaboration, this SR and MA had some limitations. First, lack of information is highlighted on the blinding of outcome assessment in some studies. Second, no additional analyses or meta-regressions were performed to explain possible sources of heterogeneity during some follow-up times $1^{2}$ values $>60 \%$. Lastly, this SR and MA did not use the GRADE (Grading of Recommendations, Assessment, Development and Evaluation) methodology to evaluate the degrees of recommendation of the studies selected. Nonetheless, the evaluation of the risk of bias de los RCTs showed that most of the studies included had low risk of bias for the principal domains of the Cochrane RoB 1 tool.
In conclusion, this study demonstrates the protective effect of the educational interventions in adult patients with HF, compared with usual care, to reduce readmissions and days of hospital stay due to decompensation of the disease. Additionally, the results can be useful to reaffirm the need to implement in the clinical practice these intervention strategies during broad followup periods and which approach the patient during the transition from hospital to the home. Finally, the importance of participation of nurses in the multidisciplinary teams for the therapeutic approach of adult patients with HF is evident.

Acknowledgments: the authors thank Mónica López de Ávila for her support during the first stage of the research.

Aid and funding: this article derives from the research "Meta-analysis of the Effect of Educational Interventions on Knowledge and Selfcare Behaviors to Improve Clinical Outcomes in Patients with Heart Failure", project funded by the research development committee (CODI, for the term in Spanish) at Universidad de Antioquia (2018-22430). 


\section{References}

1. Imizcoz MÁ. Insuficiencia cardíaca. Definición, fisiopatología y cambios estructurales. Cir. Cardiov. 2008;15(1):15-20.

2. Pons F, Lupón J, Urrutia A, González B, Crespo E, Díez C, et al. Mortalidad y causas de muerte en pacientes con insuficiencia cardiaca: experiencia de una unidad especializada multidisciplinaria. Rev. Esp. Cardiol. 2010; 63(3):303-14.

3. Gómez E. Capítulo 2. Introducción, epidemiología de la falla cardiaca e historia de las clínicas de falla cardiaca en Colombia. Rev. Colomb. Cardiol. 2016;23(Suppl 1):6-12.

4. Caballero A, Pinilla MI, Mendoza ICS, Peña JRA. Frecuencia de reingresos hospitalarios y factores asociados en afiliados a una administradora de servicios de salud en Colombia. Cad. Sau de Pu blica. 2016; 32(7):e00146014.

5. Farmakis D, Papingiotis G, Parissis J. Acute heart failure: Epidemiology and socioeconomic burden. Contin. Cardiol. Educ. 2017; 3(3):88-92.

6. Augustovski F, Caporale J, Fosco M, Alcaraz A, Diez M, Thierer J, et al. Uso de recursos y costos de hospitalizaciones por insuficiencia cardíaca: un estudio retrospectivo multicéntrico en Argentina. Value Health Reg. Issues. 2017; 14:73-80.

7. Moher D, Liberati A, Tetzlaff J, Altman DG. Preferred Reporting Items for Systematic Reviews and Meta-Analyses: The PRISMA Statement. PLoS Med. 2009; 6(7):e1000097.

8. Higgins J, Thomas J, Chandler J, Cumpston M, Li T, Page M, et al. Cochrane Handbook for Systematic Reviews of Interventions version 6.2. 2021 [cited 2021 Jan 11]. Available from: https://training.cochrane.org/cochranehandbook-systematic-reviews-interventions

9. Higgins JPT, Altman DG, Gøtzsche PC, Jüni P, Moher D, Oxman AD, et al. The Cochrane Collaboration's tool for assessing risk of bias in randomised trials. BMJ. 2011; 343:d5928.

10. Aldamiz-Echevarria B, Muñiz J, Rodríguez-Fernández JA, Vidan-Martinez L, Silva-Cesar M, Lamelo-Alfonsin F, et al. Ensayo clínico aleatorizado y controlado para valorar una intervención por una unidad de hospitalización domiciliaria en la reducción de reingresos y muerte en pacientes dados de alta del hospital tras un ingreso por insuficiencia cardiaca. Rev. Esp. Cardiol. 2007; 60(9):914-22.

11. Atienza F, Anguita M, Martinez-Alzamora N, Osca J, Ojeda S, Almenar L, et al. Multicenter randomized trial of a comprehensive hospital discharge and outpatient heart failure management program. Eur. J. Heart Fail. 2004; 6(5):643-52.

12. Blue L, Lang E, McMurray JJ, Davie AP, McDonagh TA, Murdoch DR, et al. Randomised controlled trial of specialist nurse intervention in heart failure. BMJ. 2001; 323(7315):715-8.

13. Boyde M, Peters R, New N, Hwang R, Ha T, Korczyk D. Self-care educational intervention to reduce hospitalisations in heart failure: A randomised controlled trial. Eur. J. Cardiovasc. Nurs. 2018; 17(2):178-85.

14. Jack BW, Chetty VK, Anthony D, Greenwald JL, Sanchez GM, Johnson AE, et al. A reengineered hospital discharge program to decrease rehospitalization. Ann. Intern. Med. 2009; 150(3):178-87.

15. Brotons C, Falces C, Alegre J, Ballarín E, Casanovas J, Catà T, et al. Ensayo clínico aleatorizado para evaluar la efectividad de una intervención domiciliaria en pacientes con insuficiencia cardiaca: estudio IC-DOM. Rev. Esp. Cardiol. 2009; 62(4):400-8.

16. Cañon-Montañez W, Oróstegui-Arenas M. Intervenciones educativas de enfermería en pacientes ambulatorios con falla cardiaca. Enferm. Glob. 2013; 12(31):52-67.

17. Cui X, Zhou X, Ma L, Sun T, Bishop L, Gardiner FW, et al. A nurse-led structured education program improves self-management skills and reduces hospital readmissions in patients with chronic heart failure: a randomized and controlled trial in China. Rural Remote Health. 2019; 19(2):5270.

18. Davis KK, Mintzer M, Dennison Himmelfarb CR, Hayat MJ, Rotman S, Allen J. Targeted intervention improves knowledge but not self-care or readmissions in heart failure patients with mild cognitive impairment. Eur. J. Heart Fail. 2012; 14(9):1041-9.

19. De Souza EN, Rohde LE, Ruschel KB, Mussi CM, Da-Silva L, Biolo A, et al. A nurse-based strategy reduces heart failure morbidity in patients admitted for acute decompensated heart failure in Brazil: The HELEN-II clinical trial. Eur. J. Heart Fail. 2014; 16(9):1002-8. 
20. DeBusk RF, Miller NH, Parker KM, Bandura A, Kraemer HC, Cher DJ, et al. Care management for low-risk patients with heart failure: A randomized, controlled trial. Ann. Intern. Med. 2004; 141(8):606-13.

21. Delaney C, Apostolidis B, Bartos S, Morrison H, Smith L, Fortinsky R. A Randomized Trial of Telemonitoring and Self-Care Education in Heart Failure Patients Following Home Care Discharge. Home Health Care Manag. Pract. 2013; 25(5):187-95.

22. DeWalt DA, Malone RM, Bryant ME, Kosnar MC, Corr KE, Rothman RL, et al. A heart failure self-management program for patients of all literacy levels: A randomized, controlled trial [ISRCTN11535170]. BMC Health Serv Res. 2006; 6:30.

23. Domingues FB, Clausell N, Aliti GB, Dominguez DR, Rabelo ER. Education and telephone monitoring by nurses of patients with heart failure: randomized clinical trial. Arq. Bras. Cardiol. 2011; 96(3):233-9.

24. Doughty RN, Wright SP, Pearl A, Walsh HJ, Muncaster S, Whalley GA, et al. Randomized, controlled trial of integrated heart failure management: The Auckland Heart Failure Management Study. Eur. Heart J. 2002; 23(2):139-46.

25. Dracup K, Moser DK, Pelter MM, Nesbitt TS, Southard J, Paul SM, et al. Randomized, controlled trial to improve self-care in patients with heart failure living in rural areas. Circulation. 2014; 130(3):256-64.

26. Ducharme A, Doyon O, White M, Rouleau JL, Brophy JM. Impact of care at a multidisciplinary congestive heart failure clinic: a randomized trial. CMAJ. 2005; 173(1):40-5.

27. Gámez-López AL, Bonilla-Palomas JL, Anguita-Sánchez M, Castillo-Domínguez JC, Arizón del Prado JM, Suárez de Lezo $\mathrm{J}$. Efectos sobre la mortalidad y reingresos hospitalarios de tres tipos distintos de programas de intervención en pacientes hospitalizados por insuficiencia cardiaca: ensayo clínico aleatorizado. Med. Clin (Barc). 2012; 138(5):192-8.

28. González-Guerrero JL, Alonso-Fernández T, García-Mayolín N, Gusi N, Ribera-Casado JM. Effectiveness of a followup program for elderly heart failure patients after hospital discharge. A randomized controlled trial. Eur. Geriatr. Med. 2014; 5(4):252-7.

29. Hägglund E, Lyngå P, Frie F, Ullman B, Persson H, Melin M, et al. Patient-centred home-based management of heart failure: Findings from a randomised clinical trial evaluating a tablet computer for self-care, quality of life and effects on knowledge. Scand. Cardiovasc. J. 2015; 49(4):193-9.

30. Holland R, Brooksby I, Lenaghan E, Ashton K, Hay L, Smith R, et al. Effectiveness of visits from community pharmacists for patients with heart failure: HeartMed randomised controlled trial. BMJ. 2007; 334(7603):1098.

31. Jaarsma T, Halfens R, Abu-Saad HH, Dracup K, Gorgels T, van Ree J, et al. Effects of education and support on self-care and resource utilization in patients with heart failure. Eur. Heart J. 1999; 20(9):673-82.

32. Jaarsma T, Wal MH, Lesman-Leegte I, Luttik ML, Hogenhuis J, Veeger NJ et al. Effect of moderate or intensive disease management program on outcome in patients with heart failure: Coordinating Study Evaluating Outcomes of Advising and Counseling in Heart Failure (COACH). Arch. Intern. Med. 2008; 168(3):316-24.

33. Jerant AF, Azari R, Nesbitt TS. Reducing the cost of frequent hospital admissions for congestive heart failure: a randomized trial of a home telecare intervention. Med. Care. 2001; 39(11):1234-45.

34. Kato NP, Kinugawa K, Sano M, Kogure A, Sakuragi F, Kobukata K, et al. How effective is an in-hospital heart failure self-care program in a Japanese setting? Lessons from a randomized controlled pilot study. Patient Prefer. Adherence. 2016; 10:171-81.

35. Kimmelstiel C, Levine D, Perry K, Patel AR, Sadaniantz A, Gorham N, et al. Randomized, controlled evaluation of short- and long-term benefits of heart failure disease management within a diverse provider network: The SPANCHF trial. Circulation. 2004; 110(11):1450-5.

36. Koelling TM, Johnson ML, Cody RJ, Aaronson KD. Discharge education improves clinical outcomes in patients with chronic heart failure. Circulation. 2005; 111(2):179-85.

37. Krumholz HM, Amatruda J, Smith GL, Mattera JA, Roumanis SA, Radford MJ, et al. Randomized trial of an education and support intervention to prevent readmission of patients with heart failure. J. Am. Coll. Cardiol. 2002; 39(1):83-9.

38. Leventhal ME, Denhaerynck K, Brunner-La Rocca HP, Burnand B, Conca-Zeller A, Bernasconi AT, et al. Swiss Interdisciplinary Management programme for Heart Failure (SWIM-HF): a randomised controlled trial study of an outpatient inter-professional management programme for heart failure patients in Switzerland. Swiss Med. Wkly. $2011 ; 141:$ w13171.

39. Mau MKLM, Lim E, Kaholokula JK, Loui TMU, Cheng Y, Seto TB. A randomized controlled trial to improve heart failure disparities: The Mālama Pu‘uwai (caring for heart) Study. Open Access J. Clin. Trials. 2017; 9:65-74. 
40. Melin M, Hägglund E, Ullman B, Persson H, Hagerman I. Effects of a Tablet Computer on Self-care, Quality of Life, and Knowledge: A Randomized Clinical Trial. J. Cardiovasc. Nurs. 2018; 33(4):336-43.

41. Naylor MD, Brooten DA, Campbell RL, Maislin G, McCauley KM, Schwartz JS. Transitional care of older adults hospitalized with heart failure: a randomized, controlled trial. J. Am. Geriatr. Soc. 2004; 52(5):675-84.

42. Negarandeh R, Zolfaghari M, Bashi N, Kiarsi M. Evaluating the Effect of Monitoring through Telephone (TeleMonitoring) on Self-Care Behaviors and Readmission of Patients with Heart Failure after Discharge. Appl. Clin. Inform. 2019; 10(2):261-8.

43. Otsu $\mathrm{H}$, Moriyama M. Effectiveness of an educational self-management program for outpatients with chronic heart failure. Jpn. J. Nurs. Sci. 2011; 8(2):140-52.

44. Ramachandran K, Husain N, Maikhuri R, Seth S, Vij A, Kumar M, et al. Impact of a comprehensive telephonebased disease management programme on quality-of-life in patients with heart failure. Natl. Med. J. India. 2007; 20(2):67-73.

45. Rodríguez-Gázquez MA, Arredondo-Holguín E, Herrera-Cortés R. Efectividad de un programa educativo en enfermería en el autocuidado de los pacientes con insuficiencia cardíaca: ensayo clínico controlado. Rev. LatinoAm. Enfermagem. 2012; 20(2):296-306.

46. Ruschel KB, Rabelo-Silva ER, Rohde LE, de Souza EN, Mussi CM, Polanczyk CA. Cost-Effectiveness of a Home Visit Program for Patients with Heart Failure in Brazil: Evidence from a Randomized Clinical Trial. Value Health Reg. Issues. 2018; 17:81-7.

47. Sethares KA, Elliott K. The effect of a tailored message intervention on heart failure readmission rates, quality of life, and benefit and barrier beliefs in persons with heart failure. Heart Lung. 2004; 33(4):249-60.

48. Stewart S, Chan Y, Wong C, Jennings G, Scuffham P, Esterman A, et al. Impact of a nurse-led home and clinicbased secondary prevention programme to prevent progressive cardiac dysfunction in high-risk individuals: the Nurse-led Intervention for Less Chronic Heart Failure (NIL-CHF) randomized controlled study. Eur. J. Heart Fail. 2015; 17(6):620-30.

49. Tomita MR, Tsai B, Fisher NM, Kumar NA, Wilding G, Stanton K, et al. Effects of multidisciplinary internet-based program on management of heart failure. J. Multidiscip. Healthc. 2009; 2:13-21.

50. Tsuchihashi-Makaya M, Matsuo H, Kakinoki S, Takechi S, Kinugawa S, Tsutsui H, et al. Home-based disease management program to improve psychological status in patients with heart failure in Japan. Circ. J. 2013; 77(4):926-33.

51. Wakefield BJ, Ward MM, Holman JE, Ray A, Scherubel M, Burns TL, et al. Evaluation of home telehealth following hospitalization for heart failure: a randomized trial. Telemed. J. E. Health. 2008; 14(8):753-61.

52. Wierzchowiecki M, Poprawski K, Nowicka A, Kandziora M, Piastkowska A, Jankowiak M, et al. A new programme of multidisciplinary care for patients with heart failure in Poznań: one-year follow-up. Kardiol Pol. 2006; 64(10):1063-70.

53. Wright SP, Walsh H, Ingley KM, Muncaster SA, Gamble GD, Pearl A, et al. Uptake of self-management strategies in a heart failure management programme. Eur. J. Heart Fail. 2003; 5(3):371-80.

54. Yu DSF, Lee DTF, Stewart S, Thompson DR, Choi K, Yu C. Effect of Nurse-Implemented Transitional Care for Chinese Individuals with Chronic Heart Failure in Hong Kong: A Randomized Controlled Trial. J. Am. Geriatr. Soc. 2015; 63(8):1583-93.

55. Rice H, Say R, Betihavas V. The effect of nurse-led education on hospitalisation, readmission, quality of life and cost in adults with heart failure. A systematic review. Patient Educ. Couns. 2018; 101(3):363-74.

56. Malik AH, Malik SS, Aronow WS. Effect of home-based follow-up intervention on readmissions and mortality in heart failure patients: a meta-analysis. Future Cardiol. 2019; 15(5):377-86.

57. Takeda A, Martin N, Taylor RS, Taylor S. Disease management interventions for heart failure. Cochrane Database Syst Rev. 2019; 1(1): CD002752.

58. Cañon-Montañez W, López de Ávila M, Rodríguez-Acelas AL. Programas de autocuidado y rol de la enfermería para mejorar los resultados clínicos en pacientes con insuficiencia cardiaca. Rev. Enf. Ref. 2020; V(3):1-6.

59. Cañon-Montañez W, Duque-Cartagena T, Rodríguez-Acelas AL. Intervenciones educativas para el manejo de la falla cardiaca: estrategias para mejorar los desenlaces clínicos de los pacientes. Av. Enferm. 2021; 39(1):7-10. 